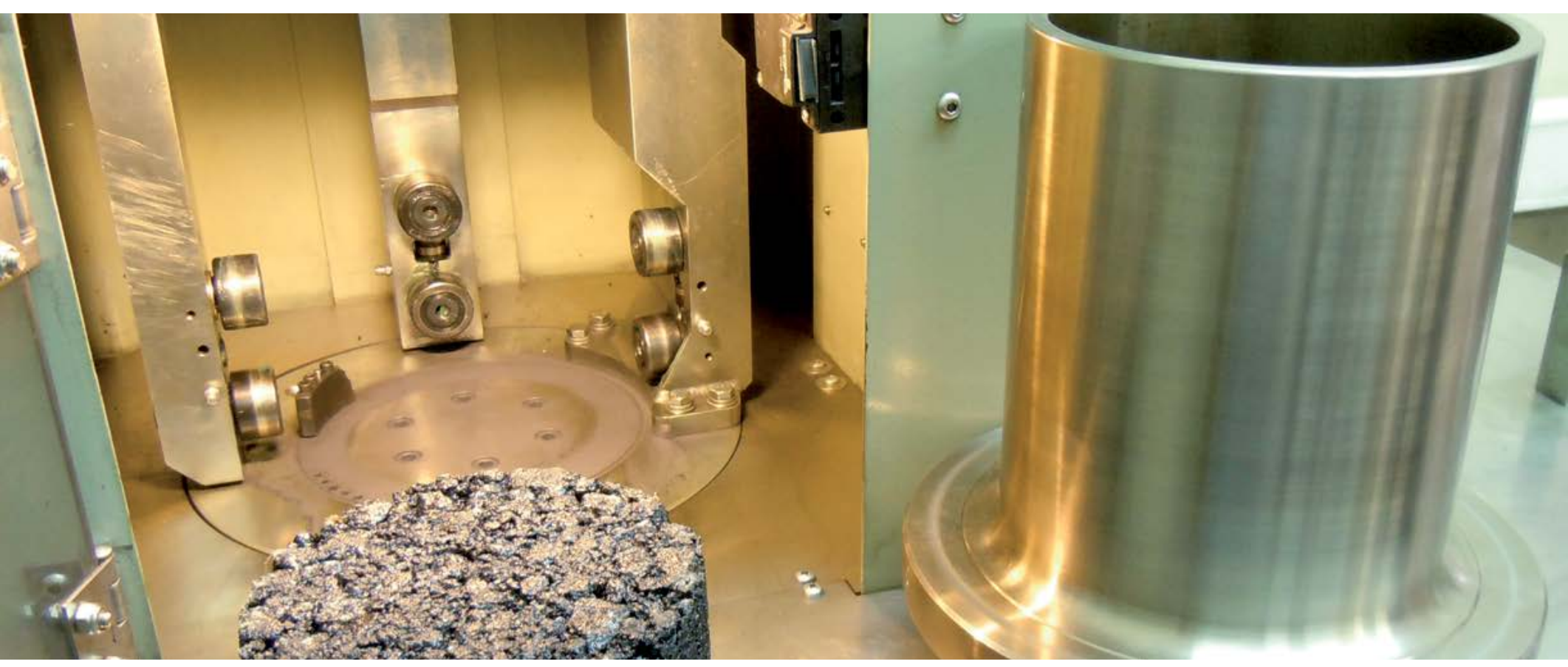

\title{
Consideraciones generales sobre la compactación de especímenes de mezcla asfáltica en caliente (MAC) en el Compactador Giratorio Superpave $\mathbb{R}$
}

Fecha de recepción: 24 de mayo del 2012

Fecha de aceptación: 10 de julio del 2012

\section{Resumen}

La compactación de especímenes de mezcla asfáltica en caliente (MAC) en el Compactador Giratorio Superpave® es uno de los desarrollos tecnológicos se diseñó con el objetivo de simular la densificación que se produce en el sitio durante la construcción y durante la vida útil. Otro objetivo fue determinar la "trabajabilidad" de la mezcla, dicho de otra manera, que tan fácil o que tan difícil resulta compactar la mezcla asfáltica. También, permitió utilizar agregados de mayor tamaño. Finalmente, el equipo debía ser lo suficientemente práctico para ser utilizado en los ensayos de aseguramiento y control de calidad. En este artículo se resumen la historia, funcionamiento del equipo, factores que afectan la uniformidad de los resultados, método de ensayo, resultados que se obtienen y finalmente, las conclusiones y recomendaciones basados en la experiencia adquirida a lo largo de los años en el LanammeUCR utilizando este equipo.
Ing. Mónica Jiménez Acuña,

Jefe del Laboratorio de Ensayos Dinámicos para Pavimentos, LanammeUCR

Tel.: (506) 2511-2513 / E-mail: monica.jimenez@ucr.ac.cr

\section{Abstract}

The compaction of hot mix asphalt (HMA) in the Superpave $®$ Gyratory Compactor is the developments in the laboratory mixture design. The compaction equipment was designed in order to simulate the densification that occurs on site during construction and in service. Another objective was to measure somehow the "workability" of the mix, said it in other words, how easy or difficult is to compact the asphalt mixture. Also, it was considered the use of larger size aggregates. Finally, the equipment should be easy enough to use in conducting the quality assurance and control tests. This article summarizes the history, the operation of the equipment, the test method, the results and finally, the conclusions and recommendations based on the experience gathered over the years using this equipment in LanammeUCR. 


\section{Historia}

Los primeros en desarrollar el concepto de la compactación giratoria fueron Philippi, Raines y Love del Departamento de Transportes de Texas, en Estados Unidos. El primer prototipo se utilizó durante los años 1939 a 1946. A partir de este prototipo, se desarrollaron dos equipos nuevos: la máquina de ensayo giratoria (Gyratory Test Machine) del Cuerpo de Ingenieros de la Armada Norteamericana y el otro por los franceses en el Laboratorio Central de Puentes y Vías de Acceso (LCPC de sus siglas en francés) llamado PGC. [Ref. 10].

En el año 1993, como parte del Programa de Investigación Estratégica en Carreteras (SHRP de sus siglas en inglés) se concibió un híbrido entre el compactador del Cuerpo de Ingenieros y la versión francesa, donde se establecieron valores para los siguientes parámetros: presión aplicada al espécimen durante la compactación, la velocidad de rotación, el número de giros aplicado y el ángulo de giro; por ejemplo, se estableció un ángulo de $1,25^{\circ}$, esto entre el valor de $6^{\circ}$ del prototipo de Texas (el cual causaba que la compactación fuera demasiado rápida, entre 15 y 18 giros) y el valor de $1^{\circ}$ del modelo francés (el cual causaba que algunas veces no se alcanzara suficiente compactación). Para este estudio se fabricaron dos modelos de compactadores giratorios: el modelo Pine Instruments Co.

AFGC125X y el Troxler Electronic Laboratories, Inc. 4140 [Ref. 10]. (Figura 1, dos imágenes de la esquina superior izquierda). Ambos modelos están en el LanammeUCR.

\section{Funcionamiento del equipo}

El Compactador Giratorio Superpave ${ }^{\circledR}$ (CGS) es un equipo que permite preparar en el laboratorio muestras de mezcla asfáltica en caliente para que luego sean ensayadas y simula la compactación con rodillo que se da en el sitio de pavimentación. El mecanismo de compactación consiste en aplicar una carga de compresión sobre la muestra a compactar, mientras se hace girar el molde con un ángulo de giro de $1,25^{\circ}$ con una velocidad de 30 revoluciones por minuto. El número de giros a aplicar depende de la cantidad de tránsito esperado en el proyecto. Los parámetros clave especificados se exponen a continuación:

Presión de compactación: $(600 \pm 18)$ kPa perpendicular al eje del molde cilíndrico que equivale a una carga de (10600 \pm 310 ) $\mathrm{N}$ en un espécimen de $150 \mathrm{~mm}$ de diámetro (Figura 2a).

Diámetro del molde: $(149,95 \pm 0,05) \mathrm{mm}$ (Figura $2 b)$.

Ángulo de giro: externo de $(1,25 \pm 0,02)^{\circ}$ e interno de $(1,16$ $\pm 0,02)^{\circ}$ (Figura 2c).

Velocidad de rotación: $(30 \pm 0,5)$ revoluciones por minuto (Figura 2d).
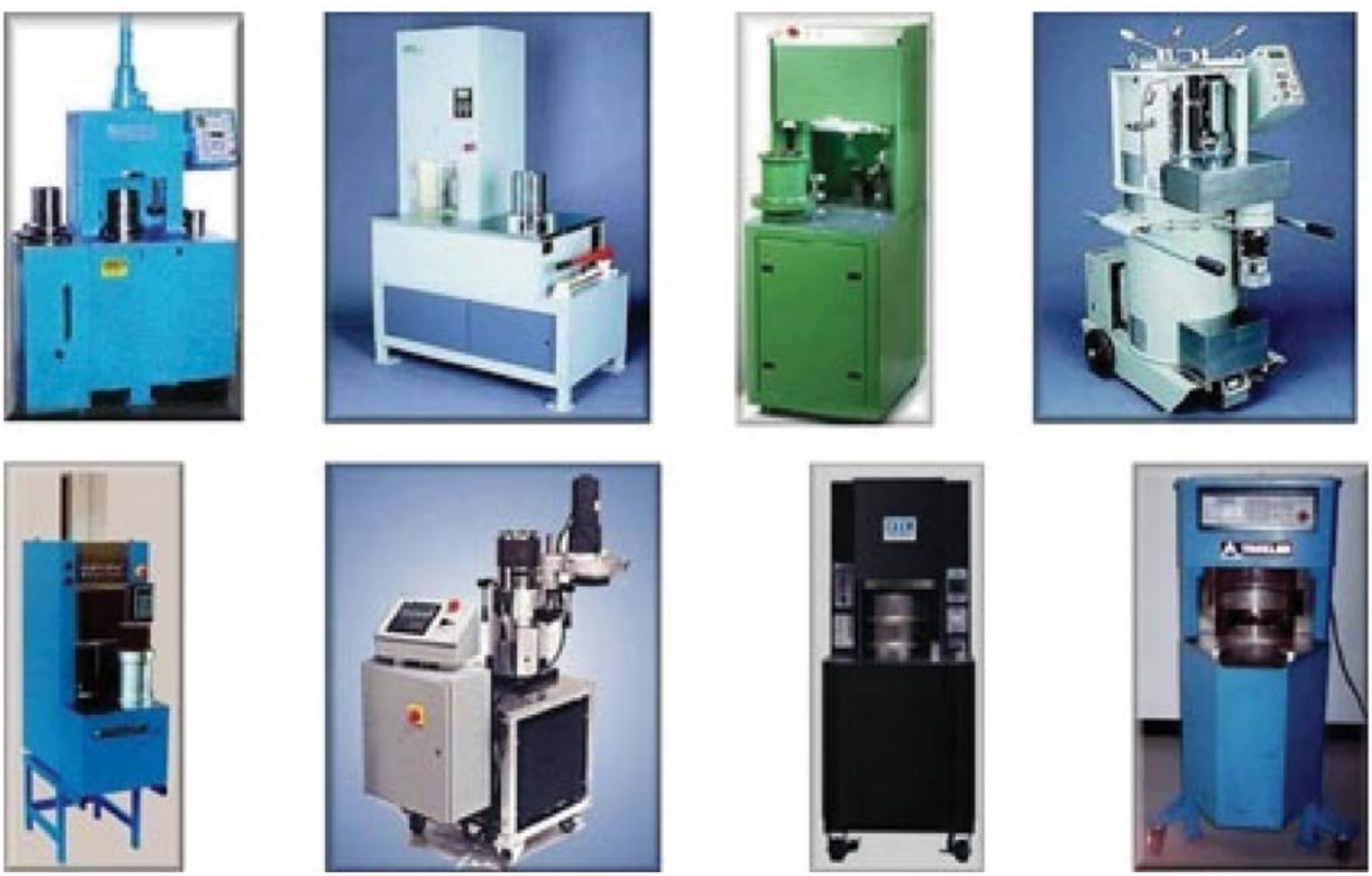

Figura 1. Varios modelos de compactadores giratorios que se encuentren en el mercado mundial.

Fuente: http://www.fhwa.dot.gov/pavement/materials/pubs/hif11301/tb00.cfm 


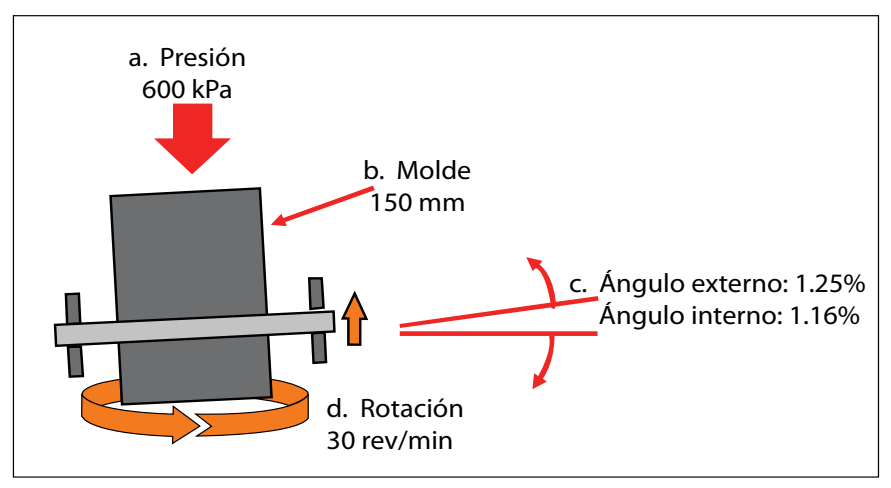

Figura 2. Esquema del funcionamiento del Compactador Giratorio Superpave ${ }^{\circledR}$

Fuente: Hot mix asphalt for seniors and graduate students. NCAT Alabama, Estados Unidos, 2004 [Ref. 12]

\subsection{Número de giros}

El número de giros a aplicar depende del diseño de mezcla, establecido para la cantidad de tránsito esperado, para lo cual se establece tres niveles de giros Ninicial, Ndiseño y Nmáximo (Figura 3).

-Ninicial: Es el número de giros utilizado para medir la capacidad de compactación de la mezcla relacionado con el proceso de construcción en sitio. Las mezclas que se compactan muy rápido (la cantidad de vacíos de aire es muy baja) son mezclas blandas, que tienden a presentar desplazamientos laterales ("shoving") durante la construcción y ser inestables bajo la acción del tránsito. Por lo tanto, se establece un valor máximo de densidad relativa, es decir, un contenido de vacíos mínimo, para evitar el uso de este tipo de mezclas.

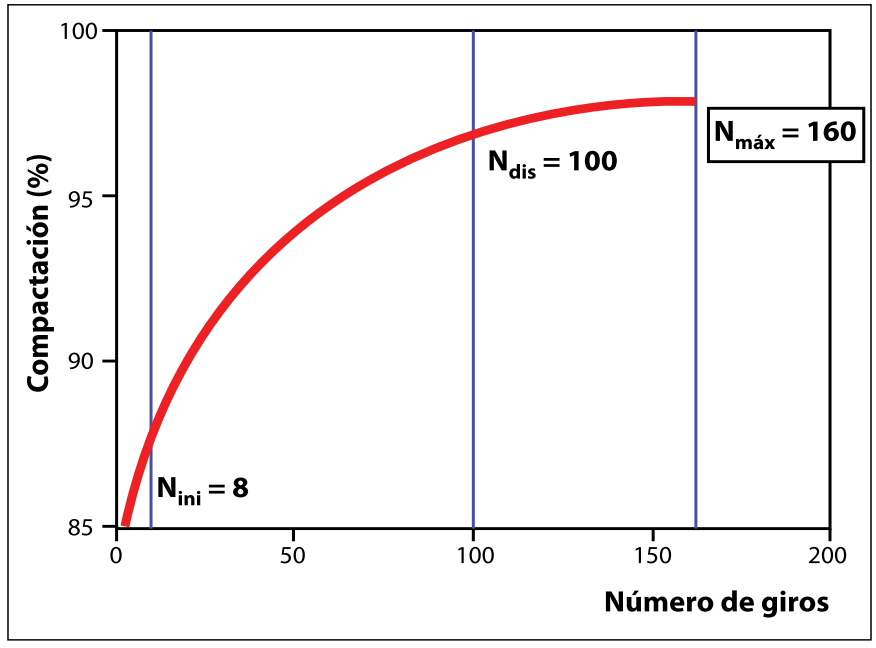

Figura 3. Comportamiento de la compactación con relación al número de giros

Fuente: http://training.ce.washington.edu/VSL/mix_design/gyratory_compactor/ gyratory_descripction.htm

-Ndiseño: Es el número de giros requeridos para producir una muestra con la misma densidad esperada en el sitio, luego de que ha pasado la cantidad de tránsito de diseño. Normalmente, se busca un $4 \%$ de vacíos de aire en el diseño de la mezcla asfáltica.

-Nmáximo: Es el número de giros para producir una densidad de laboratorio que no deber ser excedida en el sitio. Si los vacíos de aire son muy bajos, entonces la mezcla se compacta demasiado bajo la acción del tránsito que puede resultar en deformación permanente ("rutting"), de ahí que este valor no debe ser menor de $2 \%$ en el diseño de mezcla.

Tabla 1. Número de giros de compactación propuesto por SHRP

Fuente: Antecedentes del diseño y análisis de mezclas asfálticas de Superpave. Tabla V-1. 1996 [Ref. 6]

\begin{tabular}{|c|c|c|c|c|c|c|c|c|c|c|c|c|}
\hline \multirow{2}{*}{$\begin{array}{cc}\begin{array}{c}\text { ESAL's } \\
\text { diseño } \\
\left(x 10^{\circ}\right)\end{array} & \begin{array}{c}\text { Temperatura } \\
\text { máxima de } \\
\text { aire }\end{array} \\
\end{array}$} & \multicolumn{3}{|c|}{$<39^{\circ} \mathrm{C}$} & \multicolumn{3}{|c|}{$39-40^{\circ} \mathrm{C}$} & \multicolumn{3}{|c|}{$41-42^{\circ} \mathrm{C}$} & \multicolumn{3}{|c|}{$43-44^{\circ} \mathrm{C}$} \\
\hline & $\mathrm{N}_{\text {ini }}$ & $\mathrm{N}_{\text {dis }}$ & $\mathrm{N}_{\text {máx }}$ & $\mathrm{N}_{\text {ini }}$ & $\mathrm{N}_{\text {dis }}$ & $\mathrm{N}_{\text {máx }}$ & $\mathrm{N}_{\mathrm{ini}}$ & $\mathrm{N}_{\text {dis }}$ & $\mathrm{N}_{\text {máx }}$ & $\mathrm{N}_{\mathrm{ini}}$ & $\mathrm{N}_{\text {dis }}$ & $\mathrm{N}_{\text {max }}$ \\
\hline$<0,3$ & 7 & 68 & 104 & 7 & 74 & 114 & 7 & 78 & 121 & 7 & 82 & 127 \\
\hline $0,3-1$ & 7 & 76 & 117 & 7 & 83 & 129 & 7 & 88 & 138 & 8 & 93 & 146 \\
\hline $1-3$ & 7 & 86 & 134 & 8 & 95 & 150 & 8 & 100 & 158 & 8 & 105 & 167 \\
\hline $3-10$ & 8 & 96 & 152 & 8 & 106 & 169 & 8 & 113 & 181 & 9 & 119 & 192 \\
\hline $10-30$ & 8 & 109 & 174 & 9 & 121 & 195 & 9 & 128 & 208 & 9 & 135 & 220 \\
\hline $30-100$ & 9 & 126 & 204 & 9 & 139 & 228 & 9 & 146 & 240 & 10 & 153 & 253 \\
\hline$>100$ & 9 & 142 & 233 & 10 & 158 & 262 & 10 & 165 & 275 & 10 & 172 & 288 \\
\hline
\end{tabular}


En el inicio del programa SHRP (1993) se establecieron 28 niveles para la cantidad de giros a aplicar que representaban 4 regiones climáticas y 7 niveles de tránsito definido por ejes equivalentes ESAL's (Tabla 1). En el año 1999 esta tabla se redujo a 4 niveles (Tabla 2) basados en la sensibilidad de las propiedades volumétricas y rigidez de la mezcla, se eliminaron las regiones climáticas pues esto ya se tomaba en cuenta en la escogencia del asfalto por Grado de Desempeño (PG por sus siglas en inglés).

Tabla 2. Número de giros de compactación que se utiliza actualmente

Fuente: Método AASHTO R 35. Tabla 1. 2004 [Ref. 4]

\begin{tabular}{|c|c|c|c|}
\hline ESAL's diseño $\left(\mathrm{x} 10^{6}\right)$ & $\mathrm{N}_{\text {ini }}$ & $\mathrm{N}_{\text {dis }}$ & $\mathrm{N}_{\text {max }}$ \\
\hline$<0,3$ & 6 & 50 & 75 \\
\hline $0,3-<3$ & 7 & 75 & 115 \\
\hline $3-<30$ & 8 & 100 & 160 \\
\hline$\geq 30$ & 9 & 125 & 205 \\
\hline
\end{tabular}

En el año 2007, el Centro Nacional de la Tecnología del Asfalto (NCAT por sus siglas en inglés) realizó un nuevo estudio para cambiar este criterio y recomendaron que se cambiara a solo controlar el Ndis para un ángulo de giro de $1,16^{\circ}$, eliminando los conceptos de Nini y Nmáx (Tabla 3). Adicionalmente, cambiaron la consideración de tránsito a una proyección de 2 años en lugar de 20 años. Esto todavía no se ha aceptado como un cambio en la Tabla 1 de la norma de ensayo de Diseño Volumétrico Superpave ${ }^{\circledR}$ AASHTO R 35 [Ref. 4].

Tabla 3. Propuesta de cambio del NCAT

Fuente: NCHRP Reporte 573. Tabla 5.12007 [Ref 7].

\begin{tabular}{|c|c|c|c|}
\hline $\begin{array}{c}20 \text { años } \\
\text { ESAL's } \\
\text { diseño } \\
\left(\times 10^{6}\right)\end{array}$ & $\begin{array}{c}2 \text { años } \\
\text { ESAL's } \\
\text { diseño }\left(\times 10^{6}\right)\end{array}$ & $\begin{array}{c}\mathrm{N}_{\text {dis }} \text { para } \\
\text { asfaltos } \\
<P G ~ 76-X X\end{array}$ & $\begin{array}{c}N_{\text {dis }} \text { para asfaltos } \geq P G \text { 76-XX } \\
\text { o mezclas colocadas en capas } \\
\text { de 10 cm desde la superficie }\end{array}$ \\
\hline$<0,3$ & $<0,03$ & 50 & NA \\
\hline $0,3-3$ & $0,03-0,23$ & 65 & 50 \\
\hline $3-10$ & $0,23-0,925$ & 80 & 65 \\
\hline $10-30$ & $0,925-2,5$ & 80 & 65 \\
\hline$>30$ & $>2,5$ & 100 & 80 \\
\hline
\end{tabular}

\section{Método de ensayo}

La siguiente descripción es un resumen del método de ensayo AASHTO T 312-11 Método estándar para la preparación y determinación de la densidad de especímenes de mezcla asfáltica en caliente utilizando el Compactador Giratorio Superpave ${ }^{\circledR}$.

\subsection{Alcance}

Este procedimiento cubre el mezclado y la compactación de especímenes cilíndricos de mezcla asfáltica en caliente, usando el compactador giratorio Superpave. Los especímenes son utilizados para determinar las propiedades físicas y volumétricas de la MAC. Este proceso de compactación simula la densidad, orientación del agregado y características estructurales obtenidas en campo cuando se utilizan procedimientos apropiados en la colocación de la mezcla asfáltica.

En este procedimiento también se abarca la determinación de la densidad relativa de especímenes compactados en cualquier punto del proceso de compactación.

\subsection{Equipo}

Para realizar este método de ensayo se requiere como equipo (Figura 4):

- El Compactador Giratorio Superpave ${ }^{\circledR}$

- Moldes de 150,2 mm de diámetro (moldes en uso) y con una altura de al menos de $250 \mathrm{~mm}$.

- Platos base superior e inferior que van dentro de los moldes

- Mezclador mecánico con movimiento planetario

- Vertedor para evitar segregación

- Termómetros

- Balanzas

- Hornos



Figura 4. Equipo básico para la preparación de los especímenes en el CGS 


\subsection{Método resumido}

A continuación se describe de forma resumida el método estándar con algunos comentarios adicionales que se consideran útiles pero no se recomienda que se implementen estos pasos para el montaje del ensayo, definitivamente es mejor referirse directamente al método de ensayo estándar.

3.3.1 Se combina el agregado para obtener el peso del bache con la granulometría deseada, esto puede ser mediante una combinación de apilamientos o dosificación de cada tamiz. Este peso debe ajustarse para alcanzar la altura deseada mediante especímenes de prueba ya sea para especímenes de diseño o para los ensayos de desempeño.

3.3.2 Se calientan los agregados y el asfalto a la temperatura de mezclado en un horno.

3.3.3 Temperatura de mezclado: es la temperatura a la cual el asfalto alcanza una viscosidad de $(0,17 \pm 0,02)$ $\mathrm{Pa} \cdot \mathrm{s}$. Hay que tener cuidado pues esto no es válido para asfaltos modificados.

3.3.4 Una vez que se alcanza esta temperatura (se mide directamente en el asfalto con una termocupla de vástago para realizar una rápida verificación); se procede a mezclar el agregado con el contenido de asfalto, el cual se dosifica en caliente, por lo tanto el peso es sobre el agregado (PTA). Por ejemplo, cuando se dosifica el bache los pesos se toman a temperatura ambiente, entonces el bache pesa $4500 \mathrm{~g}$, cuando se pasa el agregado al tazón de mezclado, todo está caliente por lo que el peso del agregado es menor y puede llegar a ser $4490 \mathrm{~g}$. Es entonces con el peso de 4490 con el que se dosifica el asfalto, entonces si el porcentaje de asfalto es $5,5 \%$ PTA, el peso de asfalto a dosificar es $4490 \times 5,5 / 100=247,0 \mathrm{~g}$.

3.3.5 Se mezclan vigorosamente los agregados y el asfalto, en este momento se puede verificar la temperatura de mezclado con una termocupla infrarroja.

3.3.6 Una vez que se completa el mezclado se procede a acondicionar la muestra en un horno de acuerdo con la práctica estándar AASHTO R 30 [Ref. 2]. A continuación se presenta en la Tabla 4 los tipos de acondicionamiento de la mezcla.

3.3.7 Luego del acondicionamiento, se procede a compactar los especímenes a temperatura de compactación. Previamente los moldes y platos base se calientan también.
Tabla 4. Tipos de acondicionamiento para la mezcla asfáltica ${ }^{*}$ Este acondicionamiento se aplica luego de que los especímenes se han compactado

\begin{tabular}{|c|c|c|}
\hline Tipo de acondicionamiento & Temperatura $\left({ }^{\circ} \mathbf{C}\right)$ & Tiempo (h) \\
\hline Diseño de mezcla & Compactación $\pm 3^{\circ} \mathrm{C}$ & $2 \mathrm{~h} \pm 5 \mathrm{~min}$ \\
\hline Desempeño corto plazo & $135 \pm 3^{\circ} \mathrm{C}$ & $4 \mathrm{~h} \pm 5 \mathrm{~min}$ \\
\hline Desempeño largo plazo* & $85 \pm 3^{\circ} \mathrm{C}$ & $120 \mathrm{~h} \pm 30 \mathrm{~min}$ \\
\hline
\end{tabular}

3.3.8 Temperatura de compactación: es la temperatura a la cual el asfalto alcanza una viscosidad de $(0,28 \pm 0,03)$ $\mathrm{Pa} \cdot \mathrm{s}$. Hay que tener cuidado pues esto no es válido para asfaltos modificados.

Se ha observado que el criterio de equiviscosidad no aplica para asfaltos modificados pues tiende a dar temperaturas de mezclado y compactación muy altas que envejecen al asfalto. Por lo que el Instituto del Asfalto en Estados Unidos recomienda que la temperatura de mezclado no exceda los $177^{\circ} \mathrm{C}$ y que se utilicen los métodos del ángulo de fase del reómetro dinámico de cortante o el procedimiento de flujo de cortante constante (Reporte 648 de NCHRP). Finalmente, el instituto advierte que estos procedimientos no fueron aplicados para asfaltos modificados con caucho por lo que no se sabe si funcionan bien para este tipo de modificación del asfalto.

3.3.9 Para llenar el molde se utiliza el dispositivo para evitar la segregación, cuando la mezcla está en este cono, se comprueba la temperatura de compactación con la termocupla infrarroja (Figura 5) Se llena el molde con la mezcla en una sola capa. Se utiliza el aditamento tipo cono para evitar la segregación de la mezcla a la hora de llenar el molde (Figura 5).

3.3.10Se coloca el molde en el compactador giratorio donde se han programado de previo los parámetros de presión, ángulo de giro y cantidad de giros. Se tiene que registrar la altura para cada giro de compactación (Figura 5).

3.3.11Una vez que se llega al número de giros deseado el compactador se detiene y entonces se procede a desmoldar la pastilla (Figura 5).

3.3.12Una vez que se tienen los especímenes compactados a temperatura ambiente se procede a realizar la medición de la gravedad específica bruta (Gbs) de acuerdo con el método AASHTO T 166 y gravedad máxima teórica $(\mathrm{Gmm})$ de acuerdo con el método AASHTO T 209, para realizar los cálculos volumétricos. 


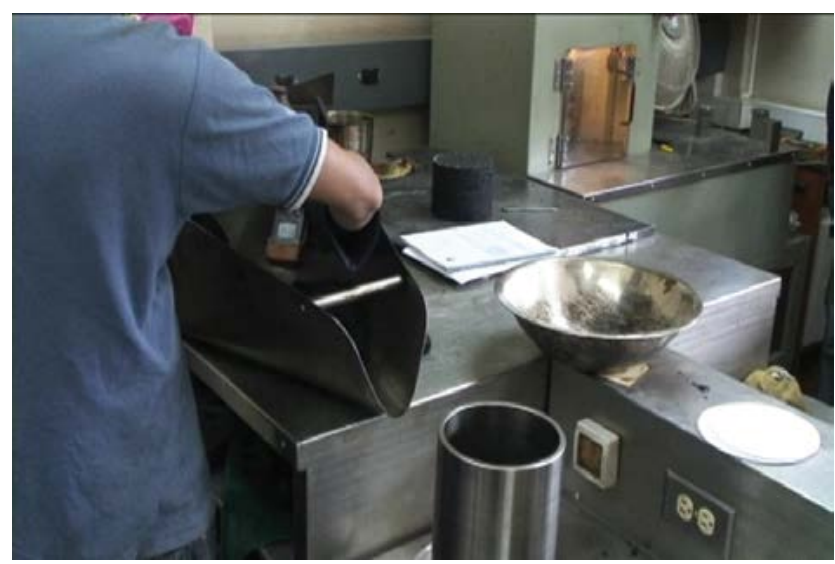

a. Temperatura de compactación

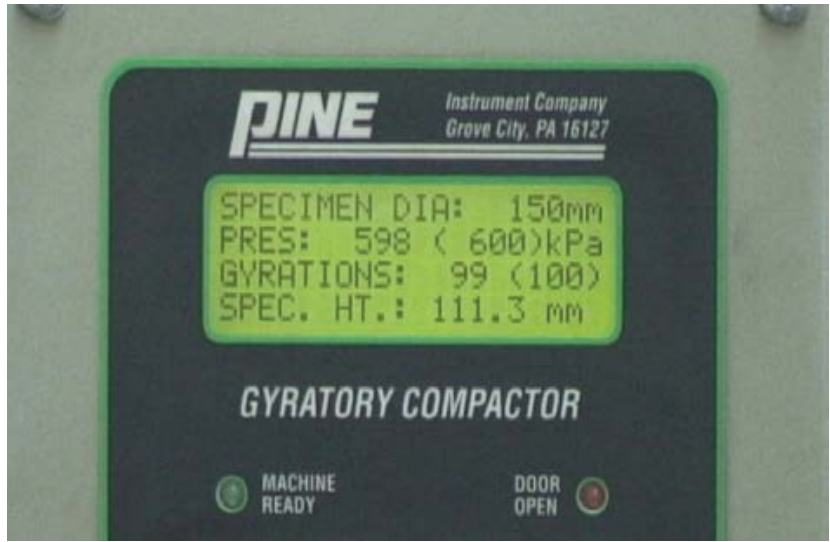

c. Parámetros de compactación

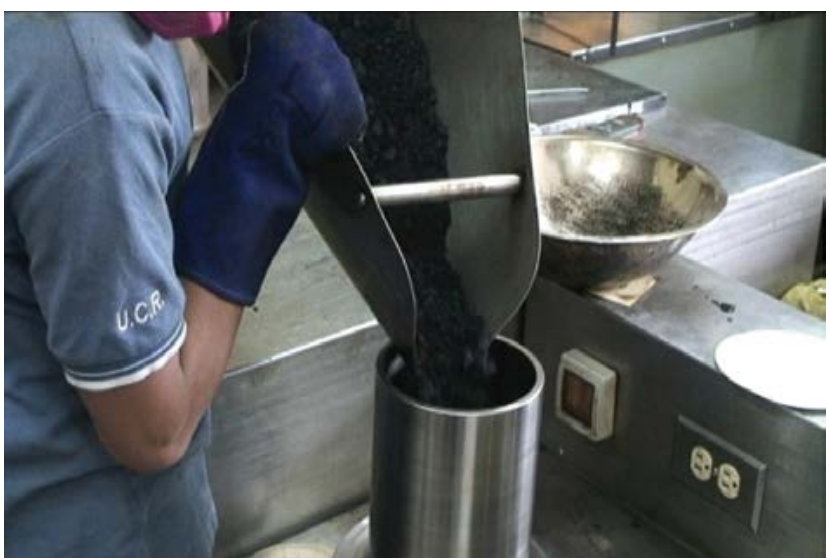

b. Llenado del molde

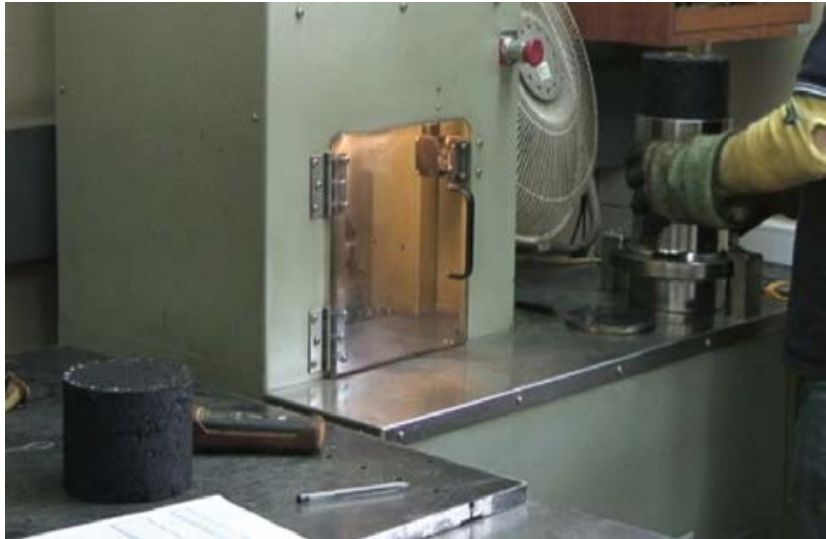

d. Desmolde de pastilla

Figura 5. Registro de temperatura de compactación y llenado de molde

\subsection{Cálculo de resultados}

Los resultados del ensayo son la densidad relativa sin corregir $\left(\% \mathrm{G}_{\mathrm{mmux}}\right)$ y la densidad relativa corregida $\left(\% \mathrm{G}_{\mathrm{mmx}}\right)$.

La densidad relativa sin corregir es un cálculo de la densidad en cualquier punto durante la compactación expresado como un porcentaje de la gravedad máxima teórica. Se le llama sin corregir debido a que la gravedad específica bruta $\left(G_{b s}\right)$ es un estimado, es decir, se basa en el cálculo entre el peso de la mezcla y el volumen del cilindro.

$$
\% \mathrm{G}_{\mathrm{mmux}}=\frac{\mathrm{W}_{\mathrm{m}}}{\mathrm{W}_{\mathrm{mx}} \mathrm{G}_{\mathrm{mm}} \mathrm{G}_{\mathrm{w}}} \cdot 100
$$

donde

$\% \mathrm{G}_{\text {mmux }}=$ densidad relativa sin corregir para cualquier giro $(\%)$

$\mathrm{W}_{\mathrm{m}}=$ peso del espécimen $(\mathrm{g})$,

$\mathrm{G}_{\mathrm{mm}}=$ gravedad específica máxima teórica de la mezcla (adimensional),

$G_{w}=$ densidad del agua $\left(1 \mathrm{~g} / \mathrm{cm}^{3}\right)$,

$\mathrm{x}=$ número de giros específico

$\mathrm{V}_{\mathrm{mx}}=$ volumen del espécimen $\left(\mathrm{cm}^{3}\right)$, que se calcula como:

$$
\mathrm{V}_{\mathrm{mx}}=\frac{\pi \mathrm{d}^{2} \mathrm{~h}_{\mathrm{x}}}{4000}
$$

donde

$\mathrm{d}$ = diámetro del espécimen ( $\mathrm{mm}$ ),

$h_{x}=$ altura del espécimen en el giro $x(m m)$

Es importante notar que la gravedad específica bruta estimada considera al espécimen como un cilindro liso, lo cual, no es real. El volumen real del espécimen es ligeramente menor, debido a la presencia de vacíos superficiales alrededor de su perímetro. Por lo tanto, de aquí surge la densidad relativa corregida, que es un factor de corrección que se obtiene de la medición directa de la gravedad específica bruta del espécimen compactado. 
$\% \mathrm{G}_{\mathrm{mmx}}=\frac{\mathrm{G}_{\mathrm{bs}} \mathrm{h}_{\mathrm{m}}}{\mathrm{G}_{\mathrm{mm}} \mathrm{h}_{\mathrm{x}}} \quad \bullet 100$ Ecuación 3

donde

$\% \mathrm{G}_{\mathrm{mmx}}=$ densidad relativa corregida (\%),

$\mathrm{G}_{\mathrm{bs}}=$ gravedad específica bruta del espécimen compactado (adimensional),

$\mathrm{h}_{\mathrm{m}}=$ altura del espécimen compactado $(\mathrm{mm})$,

$\mathrm{G}_{\mathrm{mm}}=$ gravedad específica máxima teórica de la mezcla (adimensional),

$h_{x}=$ altura del espécimen en el giro $x(m m)$,

Con los datos anteriores se pueden presentar los resultados de densificación de la mezcla, adicionalmente, la densidad relativa es uno de los parámetros más importantes en el Diseño de mezcla Superpave ${ }^{\circledR}$, la cual tiene que cumplir con un porcentaje para el Ninicial, Ndis y el Nmáx respectivamente (Tabla 5).

Tabla 5. Densidad relativa requerida en el diseño de mezcla

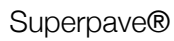

Fuente: Método AASHTO R 323. Tabla 6. 2007 [Ref. 5]

\begin{tabular}{|c|c|c|c|}
\hline \multirow{2}{*}{ ESAL's diseño $\left(x 10^{\circ}\right)$} & \multicolumn{3}{|c|}{ Densidad relativa requerida $\left(\mathrm{G}_{\mathrm{mmx}}\right)$} \\
\cline { 2 - 2 } & $\mathrm{N}_{\text {ini }}$ & $\mathrm{N}_{\text {dis }}$ & $\mathrm{N}_{\text {máx }}$ \\
\hline$<0,3$ & $\leq 91,5 \%$ & & \\
\hline $0,3-<3$ & $\leq 90,5 \%$ & \multirow{2}{*}{$96,0 \%$} & $98,0 \%$ \\
\hline $3-<30$ & $\leq 89,0 \%$ & & \\
\hline$\geq 30$ & & & \\
\hline
\end{tabular}

El LanammeUCR participa cada dos años en el programa de interlaboratorial de AMRL (Laboratorio de referencia de materiales de AASHTO) para este ensayo. Con el material enviado por AMRL se preparan dos especímenes compactados con su respectiva muestra de máxima
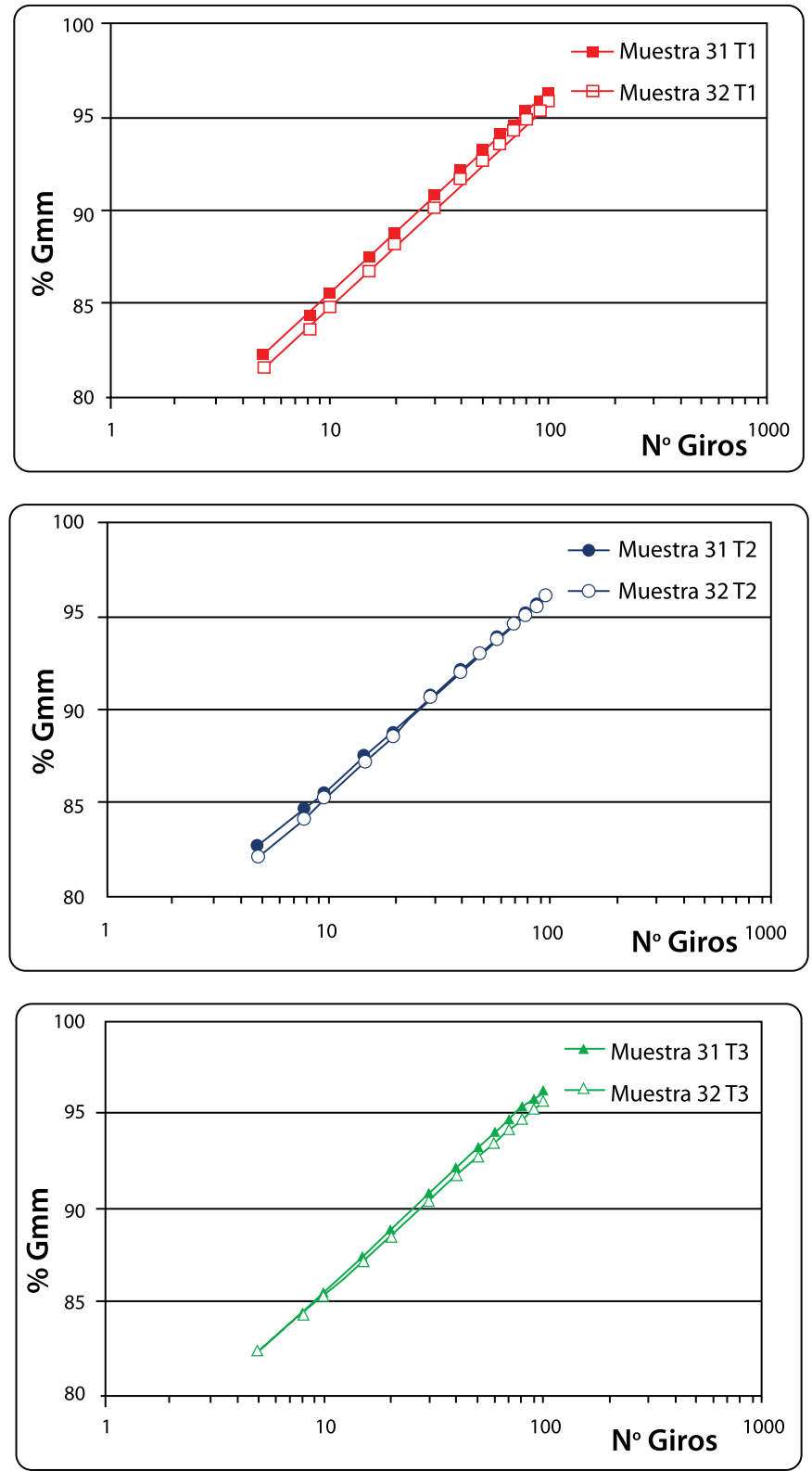

Figura 6. Curvas de densificación muestras AMRL

Tabla 6. Resumen de los resultados obtenidos en el interlaboratorial AMRL 2011

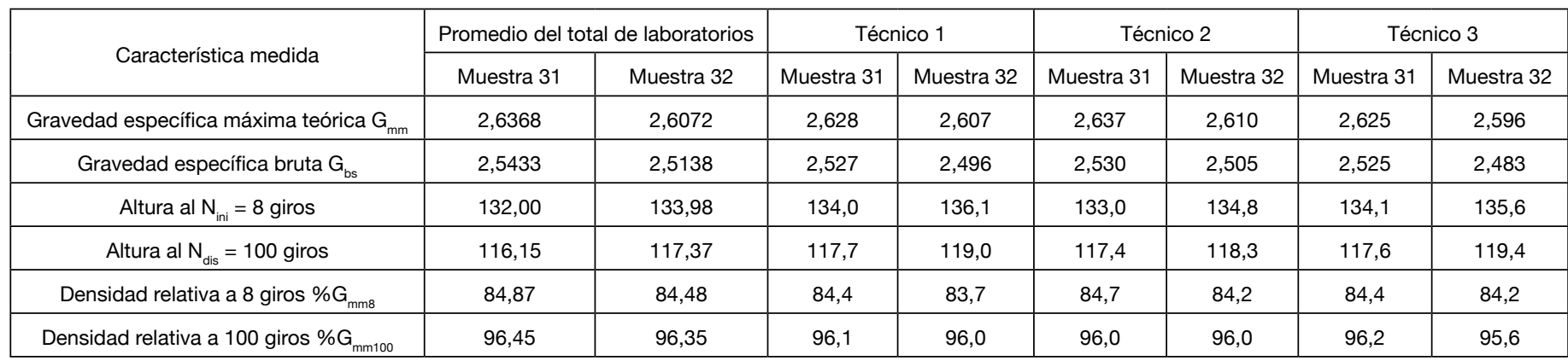


teórica. Para el año 2011, el personal técnico de laboratorio participó en el programa obteniendo resultados con altas calificaciones demostrando la competencia técnica de los técnicos en la realización de este ensayo, este año participaron 620 laboratorios. En la Tabla 6 se resumen los resultados obtenidos para cada técnico y cada muestra.

A continuación en la Figura 6 se presentan las curvas de densificación para las muestras y para cada técnico.

\subsection{Criterios de precisión y exactitud}

Los criterios de precisión son muy importantes pues permiten determinar la dispersión de los resultados obtenidos en los ensayos y ayudan a tener un criterio para aceptarlos como válidos. Con respecto a este método de ensayo es muy importante entender cómo obtuvieron estos datos, los cuales se derivaron a partir de un estudio entre 26 laboratorios, los cuales compactaron 3 especímenes de $152,6 \mathrm{~mm}$ de diámetro para dos tipos de agregado (uno de tamaño máximo nominal de $12,5 \mathrm{~mm}$ y otro de tamaño máximo nominal de 19,0 mm), con porcentajes de vacíos entre $4 \%$ y $5 \%$. El ángulo de giro externo fue el que se verificó.

Un dato muy importante que la norma destaca es que estos resultados de precisión se obtuvieron con agregados cuyas absorciones fueron menores de 1,5\%. En Costa Rica no se han encontrado agregados con absorciones tan bajas por lo que es muy importante tomar esto en cuenta a la hora de analizar los datos obtenidos, ya que a partir de la experiencia desarrollada en el LanammeUCR, en nuestro país las variaciones pueden ser mayores.

Tabla 7. Estimados de precisión para la densidad relativa. Fuente: Método AASHTO T 312, 2011 [Ref 1].

\begin{tabular}{|c|c|c|}
\hline & $\begin{array}{c}\text { Límite 1s } \\
\text { Densidad relativa (\%) }\end{array}$ & $\begin{array}{c}\text { Límite d2s } \\
\text { Densidad relativa (\%) }\end{array}$ \\
\hline \multicolumn{3}{|l|}{$\begin{array}{l}\text { Precisión un solo } \\
\text { operador: }\end{array}$} \\
\hline $\begin{array}{c}12,5 \mathrm{~mm} \text { tamaño máximo } \\
\text { nominal }\end{array}$ & 0,3 & 0,9 \\
\hline $\begin{array}{c}19,0 \text { mm tamaño máximo } \\
\text { nominal }\end{array}$ & 0,5 & 1,4 \\
\hline \multicolumn{3}{|l|}{$\begin{array}{c}\text { Precisión } \\
\text { multilaboratorio: }\end{array}$} \\
\hline $\begin{array}{c}12,5 \mathrm{~mm} \text { tamaño máximo } \\
\text { nominal }\end{array}$ & 0,6 & 1,7 \\
\hline $\begin{array}{c}19,0 \text { mm tamaño máximo } \\
\text { nominal }\end{array}$ & 0,6 & 1,7 \\
\hline
\end{tabular}

En la Tabla 7 se presentan los criterios de precisión de la norma de ensayo, la desviación estándar máxima aceptable para tres réplicas (1s) y la diferencia máxima entre las réplicas (d2s), para un mismo operador/equipo y para distintos operadores en equipos distintos.

En cuanto al criterio de exactitud no se ha definido hasta el momento un material patrón que sirva como valor de referencia por lo que no se presenta esta información.

\section{Factores que afectan la uniformidad de los resultados de la densidad}

A lo largo de los años se han reconocido varios factores que afectan la uniformidad de la densidad de los especímenes compactados en elCGS, pues anteriormente se conceptuaba que si los equipos estaban bajo control metrológico, los resultados volumétricos tenían que ser similares. A pesar de que los compactadores de distintos fabricantes están calibrados, los especímenes han presentado propiedades volumétricas distintas, lo que ha llevado a un gran debate en cuanto al ángulo de giro de compactación, debido a que la calibración de este es muy particular en cada compactador. Sin embargo, se han identificado a nivel internacional numerosas fuentes de variabilidad relacionadas con la fabricación de los especímenes en el laboratorio, las cuales se detallan a continuación.

\subsection{Mantenimiento del equipo}

Un mal estado de los componentes del compactador afecta los resultados de la medición del ángulo de giro, por lo consiguiente, también afecta los resultados volumétricos de los especímenes. Es por esto, que se debe seguir al pie de la letra, las instrucciones de mantenimiento del equipo dadas por el fabricante que incluye la frecuencia con las que se deben de realizar las tareas, por ejemplo en el modelo AFGC125XA de Pine Instruments, el fabricante recomienda que se verifique el ángulo de giro externo cada 25 horas. Adicionalmente, se tiene que tener presente que hay ciertas partes del equipo que se desgastan con el uso como los soportes, roles, etc., que se tienen que revisar de manera periódica para verificar la condición en que están, de tal manera que, si hay piezas con mucho desgaste es conveniente reemplazarlas.

Como parte del Sistema de Gestión de la Calidad del LanammeUCR, se ha establecido que todos los parámetros del equipo se calibren cada año, con verificaciones intermedias cada 6 meses (esto se puede variar de acuerdo con el uso del equipo pues si es muy intenso se recomiendan verificaciones más seguidas), es así como se asegura que 
los componentes del equipo están en óptimas condiciones de operación.

Por ejemplo, la verificación de la velocidad de giro, se hace con un cronómetro y se toma el tiempo que tarda el equipo en dar 10 revoluciones, este tiempo tiene que ser $(20,00 \pm$ $0,33) \mathrm{s}$.

Para verificar la presión se utiliza un anillo de carga calibrado para medir la fuerza del pistón sobre el anillo, iniciando con una carga de $1500 \mathrm{~N}$ e incrementando en intervalos de 1000 $\mathrm{N}$ hasta alcanzar la fuerza máxima de $18000 \mathrm{~N}$. Una vez calibrada la presión, se verifican las lecturas de altura para lo cual se utiliza un juego de bloques calibrados, a los cuales se les aplica la presión y esto se hace para distintas alturas, que se combinan configurando los bloques para obtenerlas (Figura 7).
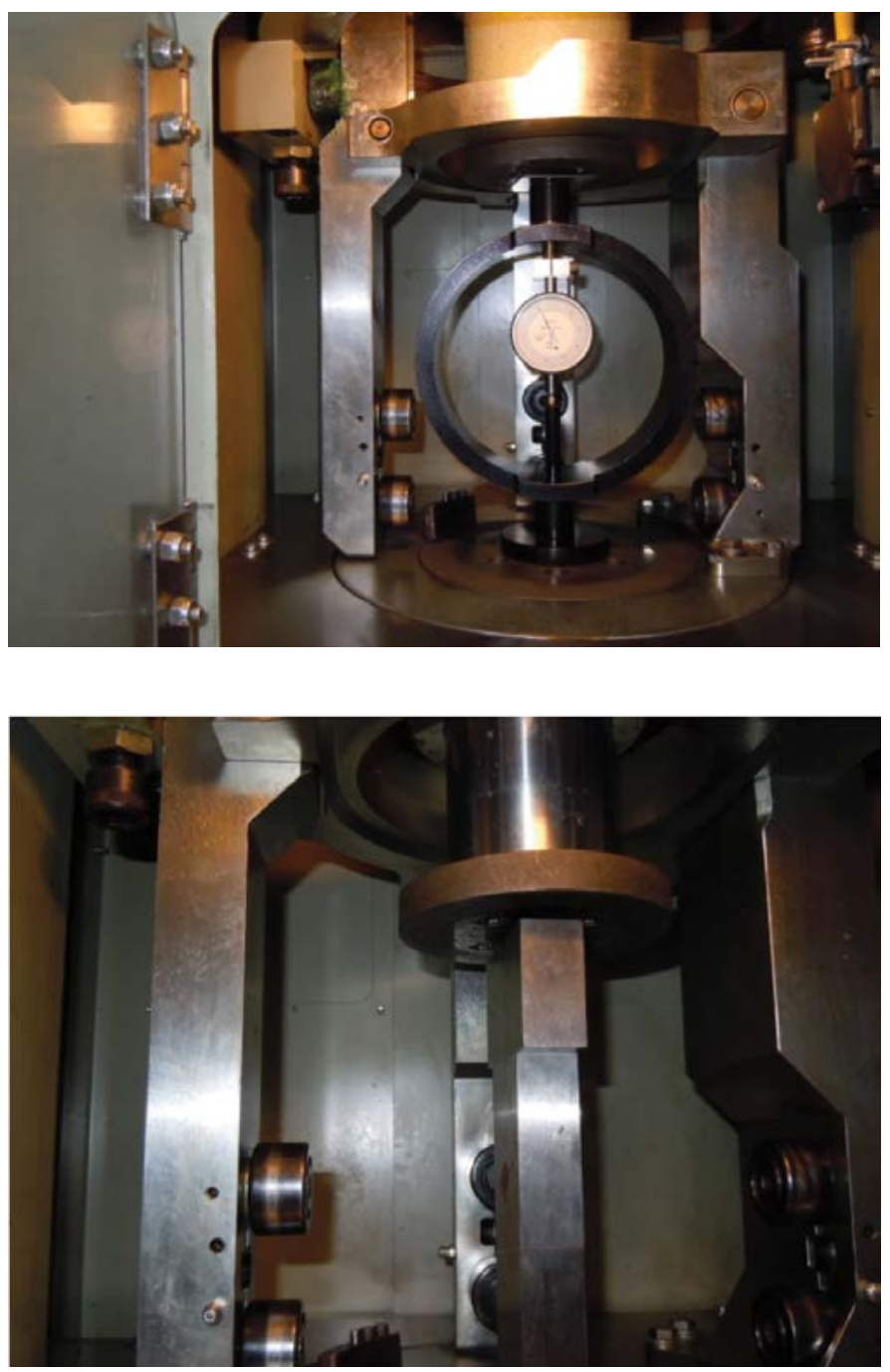

Figura 7. Calibración de la presión y altura del CGS
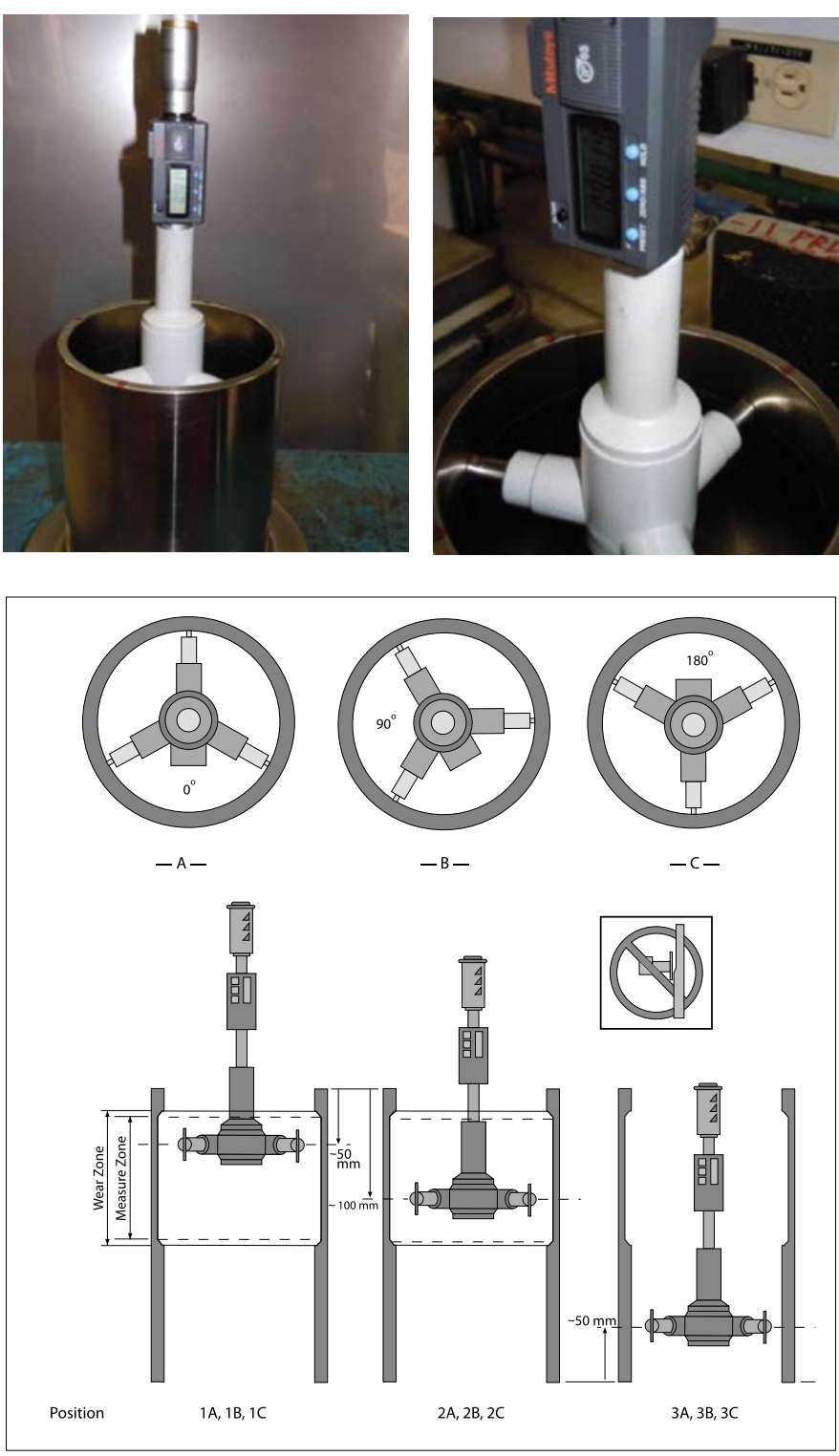

Figura 8. Medición de diámetro interno de un molde del CGS. Fuente: AASHTO T 312 Figura A3.2, 2011 [Ref. 1]

\subsection{Desgaste de los moldes de compactación}

Además de los componentes indicados anteriormente, es muy importante incluir dentro de este esquema de mantenimiento el cuidado y la revisión de los moldes de compactación, se debe asegurar la limpieza de los mismos para evitar su deterioro, y sobre todo para asegurar la uniformidad del diámetro de los especímenes. Para ello, cada año se realiza una verificación del diámetro interno de los moldes con un micrómetro con una galga de diámetros internos (Figura 8) para comprobar que el proceso de desgaste a la hora de la compactación y extracción de las pastillas no sea significativo afectando la tolerancia permitida para el diámetro. 
El desgaste del molde es mayor en el área inferior donde se da la compactación de los especímenes, por lo que se recomienda que se mida este diámetro en esa zona, entre $2,5 \mathrm{~cm}$ y $12,5 \mathrm{~cm}$ desde el fondo. El método de ensayo especifica un diámetro interno entre $149,9 \mathrm{~mm}$ a $150,0 \mathrm{~mm}$ para moldes nuevos y para moldes en uso no debe exceder $150,2 \mathrm{~mm}$.

En un estudio del 2003, Prowell y otros autores notaron que el molde tenía mucho desgaste, lo que llevó a que hubiera una luz entre las paredes del molde y los platos base lo que provocó un movimiento inusual en el plato base, que causó cambios en el ángulo interno de giro. Lo que permitió determinar que esto causa un efecto negativo en la uniformidad de las gravedades específicas pues tuvieron diferencias de hasta 0,028 en la gravedad específica bruta.

Actualmente, la norma de ensayo AASHTO T 312-11 Preparación y determinación de la densidad de especímenes de mezcla asfáltica en caliente por medio del Compactador Giratorio Superpave ${ }^{\circledR}$, incluyó en el anexo A los pasos a realizar para la evaluación del diámetro interno de los moldes pero no especifica medir la diferencia aceptable en la luz entre las paredes del molde y el plato base.

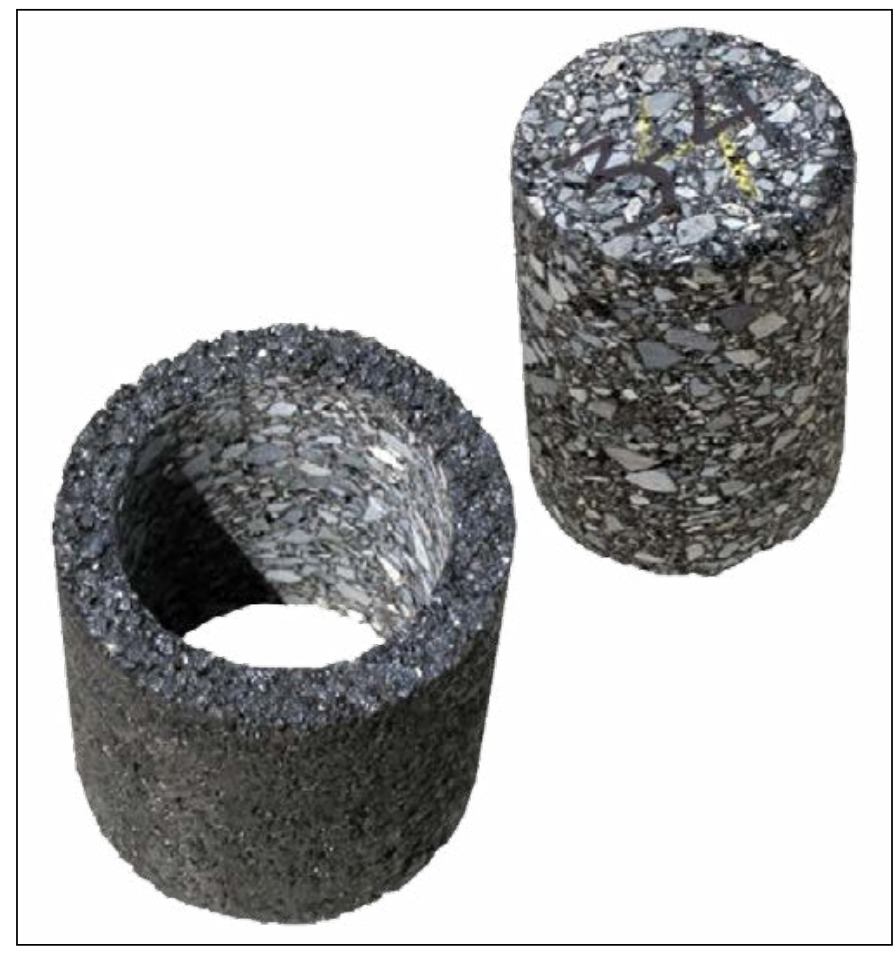

Figura 9. Núcleo extraído de un especímen compactado en el CGS. Fuente: http://training.ce.washington.edu/VSL/mix_design/gyratory_compactor/ giratory_background.htm, Figura 4, 2011 [Ref. 4]

\subsection{Limpieza del compactador}

El compactador CGS se debe mantener lo más limpio posible, esto incluye todas las superficies, roles, platos y moldes. Se ha demostrado que la "intrusión" de partículas debajo del plato base del molde tiene un efecto significativo en el ángulo de giro. Se encontró que una impureza de $0,1 \mathrm{~mm}$ de espesor puede disminuir el ángulo de giro interno efectivo en aproximadamente $0,05^{\circ}$; que dadas las especificaciones es un cambio significativo.

\subsection{Densidad no uniforme dentro de un mismo espécimen}

Los especímenes compactados en el CGS típicamente muestran una densidad no uniforme dentro del mismo espécimen, se ha logrado determinar que se desarrollan menores densidades cerca de los bordes de la pastilla. De la extracción de núcleos de $100 \mathrm{~mm}$ de diámetro de los especímenes de $150 \mathrm{~mm}$ (Figura 9), para los ensayos de desempeño SPT (Simple Performance Tests) se ha logrado determinar que tiene $1,5 \%$ menos de vacíos de aire comparado con la cantidad de vacíos del espécimen de $150 \mathrm{~mm}$ de diámetro, esta situación es bien conocida pero es aceptada.

\section{5 Ángulo de giro interno}

El ángulo externo se define como el ángulo entre la pared del molde y la vertical. El ángulo interno se define como

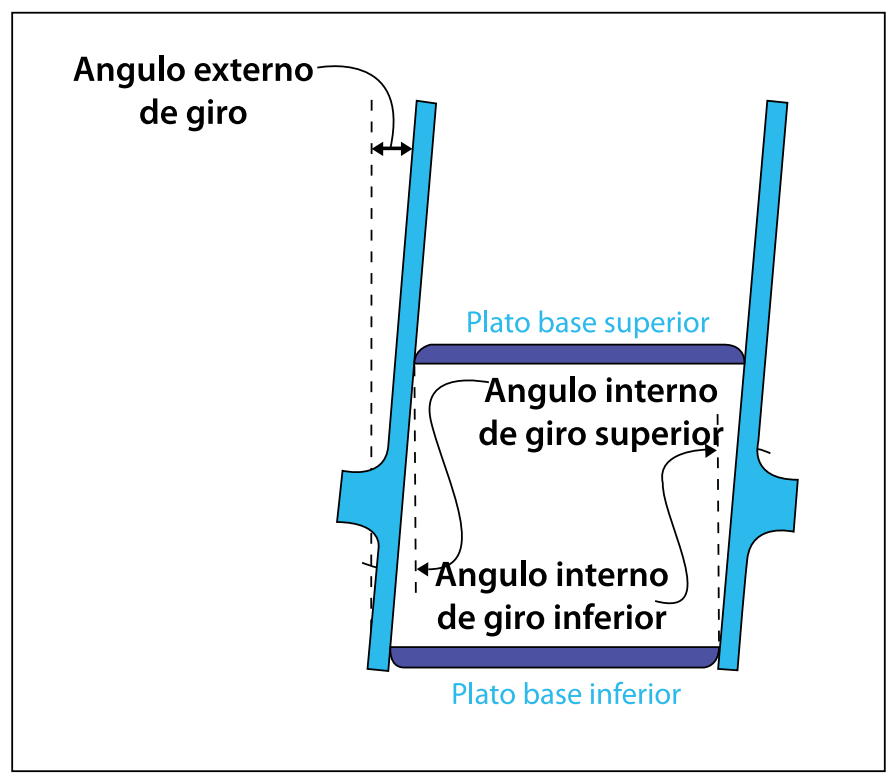

Figura 10. Ángulo interno en el CGS.

Fuente: http://training.ce.washington.edu/VSL/mix_design/gyratory_compactor/ giratory_background.htm, Figura 5, 2011 [Ref. 8] 
el ángulo entre las paredes del molde y los platos base superior e inferior (Figura 10).

Se han medido diferencias de más de $0,8 \%$ en vacíos de aire entre especímenes compactados en distintos compactadores giratorios. Estas diferencias se deben a diferencias en el ángulo interno de giro. Inicialmente el método de ensayo sólo especificaba el ángulo externo (que es más fácil de medir) pues se creía que este reproducía de manera precisa el ángulo interno de giro. Sin embargo, si los platos base superior e inferior no permanecen paralelos



Figura 11. Dispositivos de medición para el ángulo interno de giro. Fuente: $h$ ttp://www.fhwa.dot.gov/pavement/materials/pubs/hif11032/tb00.cfm, Figuras 11 y 15, 2011 [Ref. 10]

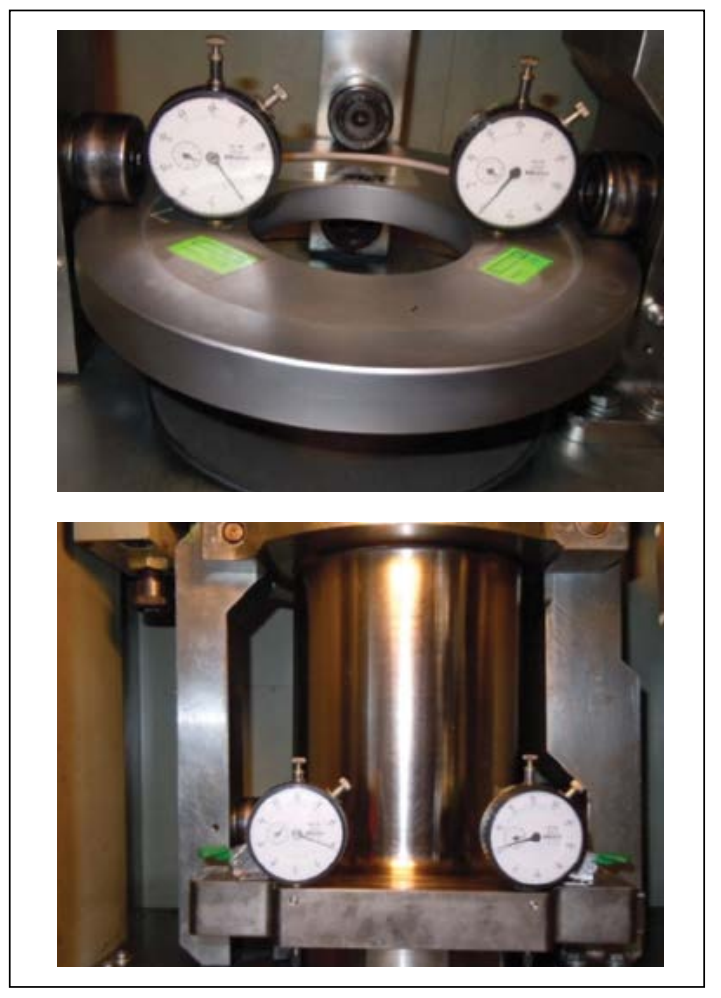

Figura 12. Medición del ángulo de giro externo del CGS. y horizontales se puede producir una diferencia significativa entre el ángulo externo e interno, con variaciones del ángulo interno entre $0,98^{\circ}$ y $1,19^{\circ}$.

En respuesta a esta situación entre los años 2003 y 2006, se han desarrollado métodos y especificaciones para validar el uso de los equipos de medición del ángulo interno bajo carga simulada.

En la actualidad existen dos equipos para medir el ángulo interno: el Medidor del Ángulo Rápido (RAM, por sus siglas en inglés) y el Simulador de Mezcla Asfáltica en caliente (HMS, por sus siglas en inglés) que se usa en conjunto con el Validador de Ángulo Dinámico (DAV, por sus siglas en inglés); los cuales no requieren de mezcla asfáltica para realizar las mediciones (Figura 11).

Actualmente, existe un método estándar AASHTO TP 71 Método estándar para la evaluación del ángulo interno de los compactadores giratorios Superpave ${ }^{\circledR}$ utilizando carga simulada.

EI LanammeUCR realiza las verificaciones del ángulo de giro externo con el dispositivo del fabricante que es un aro con 2 deformímetros separados 12,81 cm (5,042 pulg.) (Figura 12).

Se introduce el aro en el equipo y se realiza la lectura de los dos deformímetros en la posición horizontal (A1, B1), luego

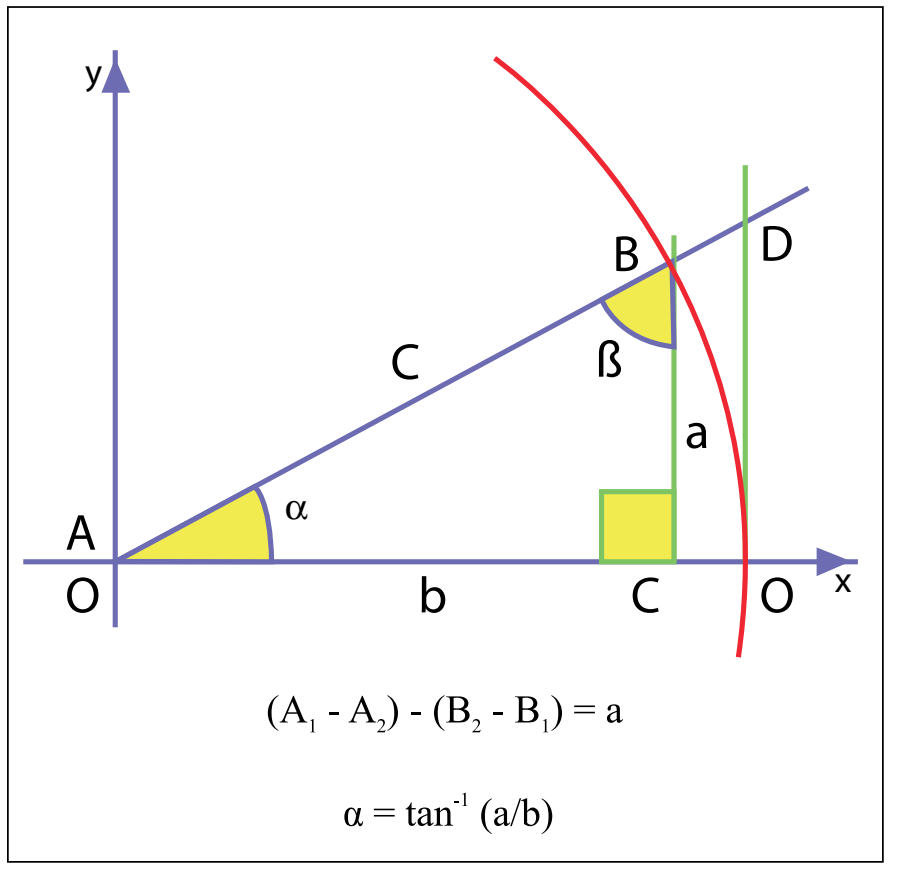

Figura 13. Cálculo del ángulo de giro externo del CGS. Fuente: Manual del CGS Pine Instruments AFGC125X, página 18, Capítulo III, 1999 [Ref. 13] 
se coloca el ángulo de giro y se realiza la medición en los dos deformímetros (A2, B2), con estas lecturas se procede a calcular el ángulo $\square$ con las ecuaciones que se muestran en la Figura 13:

Donde:

$\mathrm{A}_{1}=$ lectura del deformímetro 1 en la posición horizontal $0^{\circ}$ (pulg),

$\mathrm{A}_{2}=$ lectura del deformímetro 1 en la posición inclinada (pulg),

$\mathrm{B}_{1}=$ lectura del deformímetro 2 en la posición horizontal $0^{\circ}$ (pulg),

$\mathrm{B}_{2}=$ lectura del deformímetro 2 en la posición inclinada (pulg),

$\mathrm{a}=$ distancia del cateto opuesto,

$\mathrm{b}=$ distancia entre deformímetros, dato fijo $12,81 \mathrm{~cm}(5,042$ pulg).

Tabla 8. Criterios de precisión para los resultados AASHTO TP 71

\begin{tabular}{|c|c|c|c|c|}
\hline \multirow{2}{*}{$\begin{array}{c}\text { Rango de } \\
\text { ángulo interno } \\
\text { obtenido }\left({ }^{\circ}\right)\end{array}$} & \multicolumn{2}{|c|}{ Precisión un operador } & \multicolumn{2}{c|}{ Precisión varios operadores } \\
\cline { 2 - 5 } & Límite 1s & Límite d2s & Límite 1s & Límite d2s \\
\hline $1,014-1,290$ & 0,011 & 0,030 & 0,015 & 0,04 \\
\hline
\end{tabular}

Por ejemplo,

$$
\begin{aligned}
& \left(A_{1}-A_{2}\right)-\left(B_{1}-B_{2}\right)=a \\
& (0,3500-0,1979)-(0,3500-0,3082)=a \\
& (0,1521)-(0,0418)=a \\
& 0,1103=a
\end{aligned}
$$

Entonces para determinar el ángulo,

$\alpha=\tan ^{-1}(a / b)$

$\alpha=\tan ^{-1}(0,1103 / 5,042)$

$\alpha=1,2532^{\circ}$

El método AASHTO TP 71 establece criterios de precisión para la medición del ángulo interno basados en un estudio realizado en el año 2007, con la participación de 27 laboratorios, el cual establece lo siguiente (Tabla 8):

En el LanammeUCR se utiliza el dispositivo RAM para la verificación del ángulo interno de giro, este dispositivo utiliza un software del fabricante para el cálculo del ángulo interno superior e inferior. En la Figura 14 se presenta una imagen del software de la toma de datos.

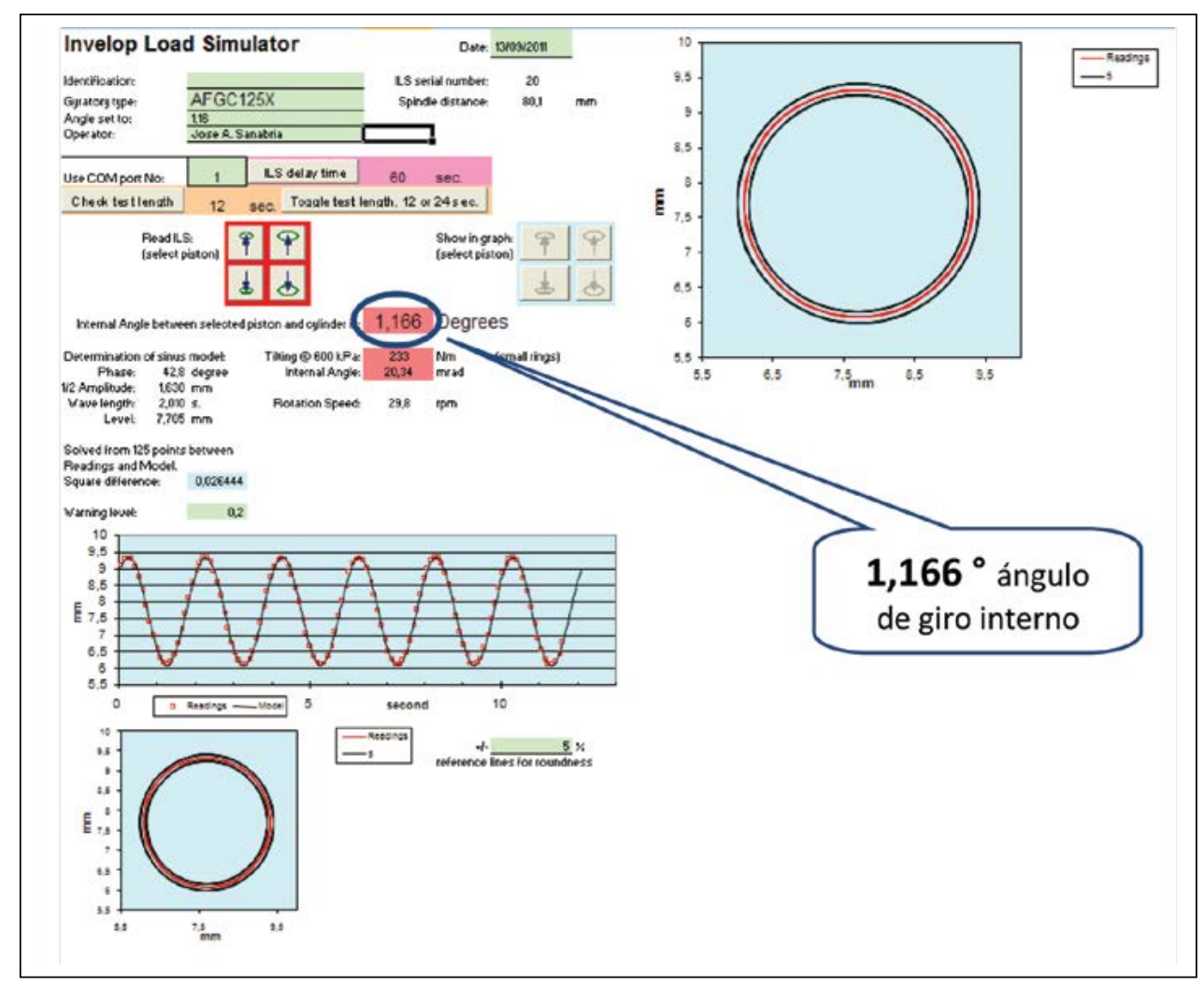

Figura 14. Medición del ángulo interno en el CGS Pine Instruments 


\section{Conclusiones y recomendaciones}

El Compactador Giratorio Superpave ${ }^{\circledR}$ fue el resultado tecnológico para simular en el laboratorio el proceso de compactación en el sitio de pavimentación, el cual simula el proceso de "amasado" que produce el rodillo sobre la mezcla asfáltica.

La densidad de los especímenes compactados en el Compactador Giratorio Superpave ${ }^{\circledR}$ puede variar debido a varios factores mencionados anteriormente por lo que es muy importante tenerlos en cuenta a la hora de implementar el método de ensayo.

El control metrológico de los dispositivos de medición o regulación del equipo es primordial para asegurar la precisión de los resultados del ensayo. Se debe poner mayor atención al ángulo de giro interno pues tener bajo control los límites al ángulo de giro externo no implica que el interno esté dentro de los parámetros de aceptación, por lo que definitivamente se recomienda que se tenga dentro del control la medición de este parámetro tan importante.

En cuanto a la preparación de la mezcla asfáltica, se debe tener especial cuidado con las temperaturas de mezclado y compactación sobre todo para asfaltos modificados donde el criterio de equiviscosidad da como resultado temperaturas muy altas que provocan más bien un envejecimiento prematuro del asfalto.

Finalmente, se recomienda la participación en una ronda de interlaboratorial para demostrar la competencia técnica en la realización del método de ensayo, pues permite que el resultado sea comparado con una cantidad importante de otros laboratorios que tienen una vasta experiencia con el ensayo.

\section{Referencias}

1. American Association of State Highway and Transportation Officials. AASHTO Designation: T 312-04, Standard method of test for preparing and determining the density of hot-mix asphalt (HMA) specimens by means of the Superpave Gyratory Compactor. Washington D.C., Estados Unidos, 27ava Edición, 2007.
2. American Association of State Highway and Transportation Officials. AASHTO Designation: $R$ 30-02, Standard practice for mixture conditioning of hot-mix asphalt (HMA). Washington D.C., Estados Unidos, 27ava Edición, 2007.

3. American Association of State Highway and Transportation Officials. AASHTO Designation: TP 71-09, Standard method of test for evaluation of Superpave Gyratory Compactor (SGC) internal angle of gyration using simulated loading. Washington D.C., Estados Unidos, 29ava Edición, 2009.

4. American Association of State Highway and Transportation Officials. AASHTO Designation: $R$ 35-04, Standard practice for Superpave volumetric design for hot-mix asphalt (HMA). Washington D.C., Estados Unidos, 27ava Edición, 2007.

5. American Association of State Highway and Transportation Officials. AASHTO Designation: M 323-07, Standard specification for Superpave volumetric mix design. Washington D.C., Estados Unidos, 27ava Edición, 2007.

6. Instituto del Asfalto. Antecedentes del diseño y análisis de mezclas asfálticas de Superpave. Kentucky, Estados Unidos, 1era Edición, 1996.

7. Prowell, B. y Brown, E.R. NCHRP Report 573: Superpave mix design: verifying gyration levels in the Ndesign table. Washington D.C., Estados Unidos, 2007.

8. National Asphalt Pavement Association. Virtual Superpave Laboratory. Educational Series 001 (ED-001). Maryland, Estados Unidos. 2005.

http://training.ce.washington.edu/VSL/about.htm

9. Asphalt Institute Technical Advisory Committee. Guidance Document: Determination of Laboratory Mixing and Compaction Temperatures for Hot-Mix Asphalt. Estados Unidos. 2011.

10. Federal Highway Administration. Technical Brief: Superpave mix design and gyratory compaction levels. FHWA HIF 11031 . Estados Unidos. 2010.

http://www.fhwa.dot.gov/pavement/materials/pubs/hif11031/tb00.cfm

11. Federal Highway Administration. Technical Brief: Review of aggregate and asphalt mixture specific gravity measurements and their impacts on asphalt mix design properties and mix acceptance. FHWA HIF $₫ 11$ 033. Estados Unidos. 2010.

http://www.fhwa.dot.gov/pavement/materials/pubs/hif11032/tb00.cfm

12. National Center of Asphalt Technology. Hot mix asphalt for seniors and graduate students. Alabama, Estados Unidos. 2004.

13. Pine Instrument Company. AFGC125XA Gyratory Compactor operation manual. Pennsylvania, Estados Unidos. 1999. 